\section{Exercise and pregnancy: focus on advice for the competitive and elite athlete}

\author{
Uğur Erdener, Richard Budgett
}

Athletes' safety is a top priority of the IOC, and the IOC Medical and Scientific Commission aims to reach evidence-based consensus on debatable topics. There are several guidelines regarding physical activity and exercise during pregnancy and postpartum period (American College of Obstetrician and Gynecologists, Canadian Society of Exercise Physiologists and Society of Obstetricians and Gynecologist of Canada, American College of Sports Medicine, The Royal College of Obstetricians and Gynecologists). However, until the IOC consensus meeting in September 2015, there was not any consensus statement on "Exercise in Pregnancy and the Post-partum Period in elite sports particularly for High-level Athletes". 1

The IOC consensus meeting had two aims: (1) to comprehensively review risk factors related to different physical and physiological states of a pregnant athlete and (2) to provide recommendations for training and competing during pregnancy and postpartum period for high levels athletes.

As IOC President Bach emphasised, "Sport is not just physical activity; it promotes health and helps prevent, or even cure, the diseases of modern civilization".

It is well known that regular exercise has tremendous health benefits, reduces risk of major illnesses and the risk of early death.

Potential health benefits of women's participation in sport are well documented. Regular physical activity and exercise helps to improve quality of life and prevent a variety of non-communicable diseases such as stroke, heart diseases, cancers or diabetes.

Profound anatomical and physiological changes accompany pregnancy but there are few reasons that pregnancy should preclude otherwise healthy women from regular exercise. "Pregnancy is recognized as a unique time for behavior modification and is no longer considered a condition for confinement."2

International Olympic Committee, Lausanne, Switzerland

Correspondence to Professor Richard Budgett, International Olympic Committee, Lausane 1007, Switzerland; richard.budgett@olympic.org
In addition, physical activity and specific exercises during pregnancy may have special benefits to the pregnant woman and fetus. Regular exercise in pregnancy provides medical and psychological benefits:

- It may reduce the occurrence of pre-eclampsia;

- Gestational diabetes mellitus risk factors such as excessive weight gain and glucose intolerance can be improved by incorporating appropriate nutrition and physical activity intervention;

- Reduce depression;

- Specific strength training of the pelvic floor muscles can prevent and treat urinary incontinence;

- It may also be useful in preventing chronic musculoskeletal conditions which could be associated with pregnancy such as low back and pelvic girdle pain.

Regular physical activity and exercise provide health benefits during the postpartum period. Regular physical activity and exercise during pregnancy is associated with improved mental health throughout gestation and the postpartum period.

\section{COMPETING IN MAJOR EVENTS WHILE PREGNANT}

It is not uncommon for pregnant women to complete in major events. We know that at least 17 athletes competed at the Olympics while pregnant. Beach Volleyball player Kerri Walsh Jennings was 5 weeks pregnant when she took her third Olympic gold medal in London 2012. Rifle shooter Nur Suryani Mohammed Taibi was 8 months pregnant at that event. Archers Khatuna Lorig (1992) and Cornelia Pfohl (2000) both won bronze medals while pregnant during Olympic Games.

There are examples of woman returning to elite competition after giving birth. Paula Radcliffe won the 2007 New York marathon just 10 months after giving birth. London Olympic gold medallist Jessica Ennis-Hill regained her Heptathlon World Championship in 2015, 13 months after giving birth.

\section{WHAT IS SAFE?}

Although in general, participation in a wide range of recreational activities is accepted to be safe, some sports with a high potential for contact, such as ice hockey, soccer, wrestling, etc, are considered to cause trauma to both the woman and fetus. Similarly, participation in some recreational activities such as gymnastics, horseback riding and downhill skiing may increase risk of falling during pregnancy. In addition, health conditions such as hypertension or intrauterine growth retardation are contraindications to exercise during pregnancy.

It has been reported that competitive athletes can maintain a more strenuous training regimen than less active women throughout pregnancy and can thus resume highintensity postpartum training sooner. Therefore, elite pregnant athletes may encounter potential risks more than recreational ones (eg, for pelvic floor dysfunctions such as urinary incontinence and pelvic organ prolapse, abdominal weaknesses and low back and pelvic girdle pain).

This meeting provided an opportunity to discuss and review exercise in pregnancy and the postpartum period in elite athletes in detail and gave us a better understanding of the specific considerations regarding participation in elite sports during pregnancy and postpartum period. There are clearly gaps in existing research on elite athletes and pregnancy. New areas of research were discussed which led to new proposals from the expert group.

Contributors UE and RB both conceived of the idea for the editorial, discussed the content and edited the final version.

Competing interests None declared.

Provenance and peer review Not commissioned; externally peer reviewed.
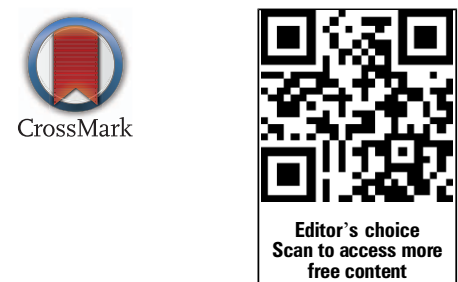

To cite Erdener U, Budgett R. Br J Sports Med 2016;50:567.

Accepted 20 December 2015

Published Online First 14 January 2016

Br J Sports Med 2016:50:567.

doi:10.1136/bjsports-2015-095680

\section{REFERENCES}

1 Barakat R, Perales M, Garatachea N, et al. Exercise during pregnancy. A narrative review asking what do we know? Br J Sports Med 2015;49: 1377-81.

2 Artal R, O'Toole M. Guidelines of the American College of Obstetricians and Gynecologists for exercise during pregnancy and the postpartum period. $\mathrm{Br} \mathrm{J}$ Sports Med 2003;37:6-12; discussion 12. 